\title{
Determination of Systolic, Mean and Diastolic Blood Pressures with Dual Cuff System is Based on Physiology
}

\author{
Jiri Jilek \\ Carditech \\ Culver City \\ California \\ USA \\ jilekj@usa.net
}

\author{
Milan Stork \\ Department of Applied Electronics and \\ Telecommunications/RICE \\ University of West Bohemia \\ Plzen, Czech Republic \\ stork@kae.zcu.cz
}

\begin{abstract}
Single cuff automatic blood pressure (BP) measurements are subject to errors because the BP values are based on statistical estimation rather than on physiological principles. We developed a dual cuff experimental system that uses an arm cuff and a wrist cuff. The wrist cuff function is similar to auscultation. This study concentrated on the determination of systolic, diastolic and mean BP using the wrist cuff pulse waveforms and the arm cuff pressures. Fifty two BP tests from our database were used to compute blood pressures using the wrist cuff method and the single arm cuff method and the two methods were compared. Mean BP values for both methods were similar but there were important individual differences. The dual cuff method should prove to be more accurate than single cuff methods because it is based on physiological principles.
\end{abstract}

Keywords - blood pressure; systolic blood pressure; mean pressure; diastolic pressure; Korotkoff sounds; dual cuff system; wrist cuff

\section{INTRODUCTION}

Single cuff automatic blood pressure (BP) monitors [1] perform most BP tests in the hospital, the clinic, and the home. Most of the monitors used today employ the method that uses pulsations in the cuff. This method is commonly called oscillometric because the pulsations in the cuff were erroneously identified as oscillations. We no longer use the term oscillometric because we identified the cuff pulsations as arterial hemodynamic pulses rather than oscillations [2].

Estimation of BP values with the single cuff method is based on statistical estimation because the method does not have physiologically identifiable points for either systolic or diastolic pressures. One of the methods, originally described by Geddes [3] uses the ratio of cuff pulse amplitudes. The method is illustrated in Fig. 1. The bottom trace represents arm cuff pulse waveforms (AW). When cuff pressure (CP)

Milan Stork's participation was supported by Department of Applied Electronics and Telecommunications, University of West Bohemia, Plzen, Czech Republic and by the European Regional Development Fund and the Ministry of Education, Youth and Sports of the Czech Republic under the Regional Innovation Centre for Electrical Engineering (RICE), project No. CZ.1.05/2.1.00/03.0094 and by the Internal Grant Agency of University of West Bohemia in Pilsen, the project SGS-2015-002 and GA15-22712S. is gradually lowered from high to low pressure the AW amplitudes increase until they reach a maximum and then continue decreasing. The point of maximum AW amplitude corresponds to mean arterial pressure (MAP). Geddes found the systolic pressure (SBP) frequently at the point of $50 \%$ of maximal $\mathrm{AW}$ amplitude and diastolic pressure (DBP) at the point of $80 \%$ of maximal amplitude. Other ratios have been used. Commercial single cuff devices use methods that are kept secret. The problems with the above described method [1] have led to the development of systems with more than one cuff. Fujikawa used custom made triple cuff [4] and Kim used two arm cuffs [5] where the second cuff is placed on the forearm. We developed an experimental dual cuff system for the determination of blood pressures and hemodynamics that uses an arm cuff and a wrist cuff. The system was first described at the AE2010 Conference [6]. The waveforms acquired from the pre-inflated wrist cuff function in a manner similar to a stethoscope used in the manual BP method.

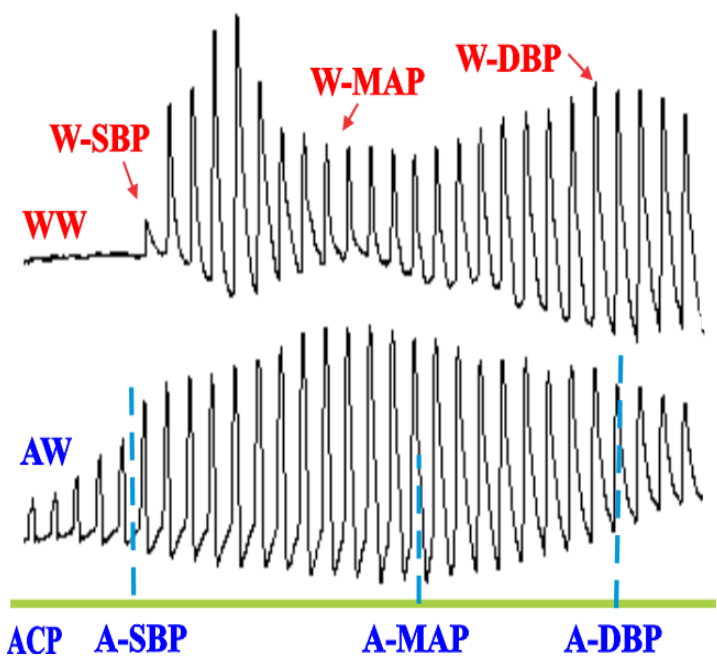

Figure 1. Gradual cuff deflation segment of a BP test. ACP is arm cuff pressure, WW are wrist cuff waveforms, AW are arm cuff waveforms, W-MAP is wrist mean pressure, A-MAP is arm cuff MAP, W-DBP is wrist cuff DBP, and A-DBP is arm cuff DBP. Segment length is $25 \mathrm{sec}$.

Fig. 1 shows wrist-cuff pulse waveforms (WW) and arm cuff pulse waveforms (AW). During gradual arm 
cuff pressure (ACP) lowering from high ACP three BP points can be determined. The point of systolic pressure (SBP) is determined as the onset of WW waveforms. This point is also known as the point where first Korotkoff sound (K1) is heard. From the SBP point the WW amplitudes quickly grow and then decrease until they reach the minimum. The wrist mean arterial pressure (W-MAP) is determined as the point of the minimum WW amplitude. The A-MAP is determined as the point of maximum AW amplitude. With further decrease of ACP the WW amplitudes increase and the AW amplitudes decrease. When the WW amplitudes no longer increase, the point of WDBP is reached. This point is known as the point where Korotkoff sounds are no longer heard. This point is known as the fifth korotkoff sound (K5). The point of A-DBP is determined using the ratio-metric method described above.

Fig. 2 shows detailed segment of WW and AW waveforms. The WW amplitudes gradually increase until they reach the W-DBP point. From W-DBP point the amplitudes plateau and the brachial artery is free of partial occlusion. The DBP value is obtained with a ratio-metric method.

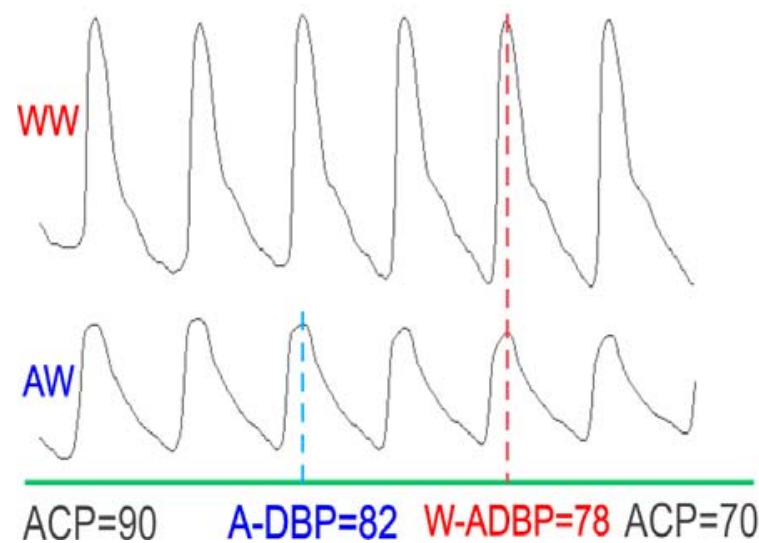

Figure 2. Detailed segment near DBP points is shown. WW are wrist cuff waveforms, AW are arm cuff waveforms, ACP is arm cuff pressure, A-DBP is arm cuff DBP and W-DBP is wrist method DBP point. Length of segment is $7.5 \mathrm{sec}$.

The main objective of this study is to show that the wrist cuff waveforms and arm cuff pressures can be used to estimate the values of SBP, DBP and MAP.

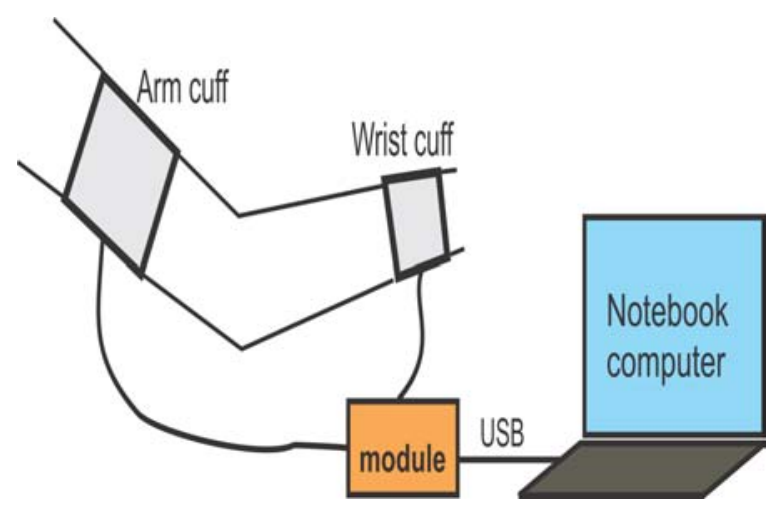

Figure 3. The dual cuff system configuration with arm cuff, wrist cuff, module, and a notebook computer.

\section{METHODS}

The dual cuff system used in this study (Fig. 3) consists of two cuffs (arm and wrist), a module and a notebook computer. Block diagram of the main module with cuffs is in Fig. 4. The two pneumatic and analog circuits for the cuffs are similar. Air pumps inflate the cuffs and cuff deflation is controlled by the valves. Piezoelectric pressure transducers (pr.xducr) provide analog signal that is amplified, filtered, and separated into two channels. One channel provides cuff pressure and the other channel provides amplified cuff-pressure waveforms. The resulting analog signals are digitized in the sub-module. Analog-to-digital conversion is 12-bit, 85 conversions/ sec operation. The digitized data are converted into USB format and made available to the notebook. The notebook contains special software that controls the module's functions and receives four channels of digitized data.

We designed the specialized software as Windowsbased multifunction system that performs data acquisition and processing functions. The function used in this study is the "Dual cuff". The dual cuff function uses the arm cuff and the wrist cuff. Both cuffs are commercial Omron cuffs. The arm cuff is an adult cuff $14 \mathrm{~cm}$ wide and the wrist cuff is $6 \mathrm{~cm}$ wide cuff. During the Dual cuff test the arm cuff is inflated to a pressure higher than expected SBP and the wrist cuff is inflated to the level of DBP.

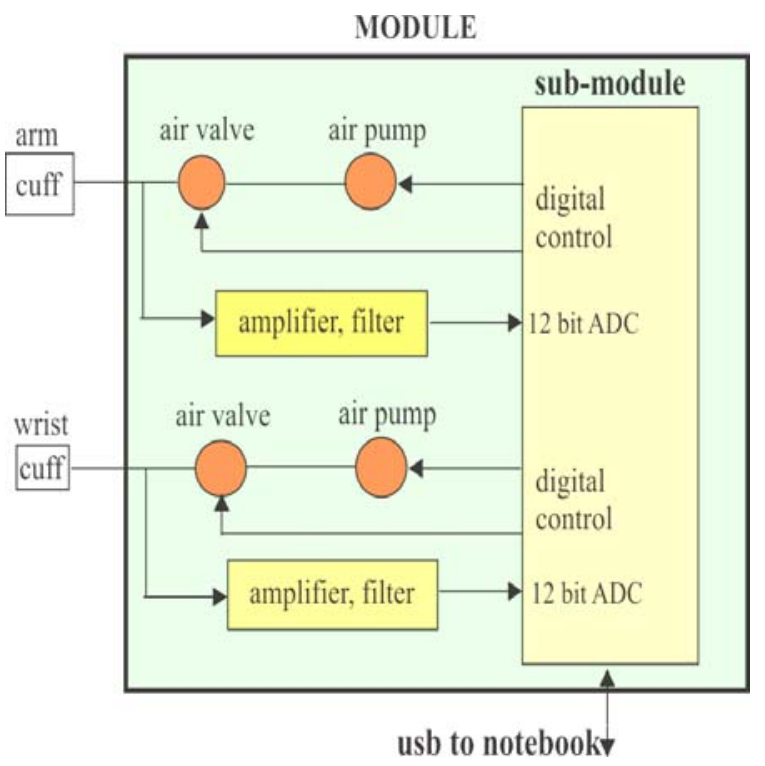

Figure 4. Block diagram of the dual cuff system's modules and the cuffs. The sub-module contains analog-to-digital conversion, control circuit, and USB interface.

Fifty two BP tests were obtained from volunteers in the sitting position, age 40-79. Six sets of data were computed from the acquired waveforms and pressures. Mean values and ranges of the computed variables are in Table 1 . W-SBP values were computed with a software routine based on first appearance of WW (Fig. 1). The values of arm cuff SBP were computed using the ratio-metric method with the amplitude ratio of 0.5. The values of arm cuff MAP (A-MAP) were computed with a software routine that determines the peak value of AW amplitudes (Fig. 1). The values of 
wrist MAP (W-MAP) were computed with a software routine that determines minimum value of WW amplitudes (Fig. 1). The values of arm cuff DBP (ADBP) were computed using ratio-metric method with the amplitude ratio of 0.76 . The values of wrist DBP (W-DBP) were computed with software routine that determined the point of beginning of the WW amplitudes plateau (Fig. 2).

\section{RESULTS}

The results in Table 1 show that the differences between the dual cuff wrist method and the single arm cuff method mean values are $3 \mathrm{mmHg}$. The W-SBP and W-MAP mean values are higher than A-SBP and A-MAP mean values. The $\mathrm{W}$-DBP mean values are lower than A-DBP mean values. The mean pulse pressure (SBP-DBP) is $57 \mathrm{mmHg}$ for the dual cuff method and $51 \mathrm{mmHg}$ for the single cuff method.

TABLE I. MEAN VALUES, STANDARD DEVIATIONS AND RANGES OF THE SIX SETS OF VALUES COMPUTED FROM WAVEFORMS AND CUFF PRESSURES. ALL VALUES ARE IN MMHG. $\mathrm{N}=52$

\begin{tabular}{|c|c|c|c|}
\hline PRESSURES & MEAN & SD & RANGE \\
\hline W-SBP & 138 & 21.2 & $112-205$ \\
\hline A-SBP & 135 & 25.8 & $116-195$ \\
\hline W-MAP & 106 & 12 & $88-138$ \\
\hline A-MAP & 103 & 15.8 & $77-150$ \\
\hline W-DBP & 81 & 9,6 & $66-104$ \\
\hline A-DBP & 84 & 15.8 & $68-103$ \\
\hline
\end{tabular}

Mean arterial pressure (MAP) is frequently calculated using the formula

$$
\mathrm{MAP}=\mathrm{DBP}+\mathrm{m} *(\mathrm{SBP}-\mathrm{DBP})
$$

where $\mathrm{m}$ is the multiplier determined from direct measurement of MAP. Traditional multiplier used in many studies is $m=0.33$. Meany et al [7] developed a more accurate multiplier $\mathrm{m}=0.41$. Table 2 shows MAP values determined from wrist waveforms and calculated MAP values using the multiplier values 0.33 (C-MAP1) and 0.41 (C-MAP2).

TABLE II. W-SBP，W-DBP，W-MAP，C-MAP1 AND C-MAP2 VALUES.

\begin{tabular}{|c|c|c|c|c|}
\hline W-SBP & W-DBP & W-MAP & C-MAP1 & C-MAP2 \\
\hline 138 & 81 & 106 & 100 & 105 \\
\hline
\end{tabular}

TABLE III. W-SBP，W-DBP，W-MAP， C-MAP1 AND C-MAP2 VALUES

\begin{tabular}{|c|c|c|c|c|}
\hline A-SBP & A-DBP & A-MAP & C-MAP1 & C-MAP2 \\
\hline 135 & 84 & 103 & 101 & 105 \\
\hline
\end{tabular}

Table 3 shows MAP values determined from arm cuff waveforms and calculated MAP values using the multipliers 0.33 (C-MAP1) and 0.41 (C-MAP2). Example of location of arm cuff and wrist cuff during blood pressure measuring is shown in Fig. 5.

\section{DISCUSSION}

We used data from a group of older volunteers because they have higher prevalence of hypertension. Hypertension is of greater interest to clinicians and researchers than normal BP values. Clinical utility of the dual cuff method can be demonstrated by calculating Isolated Systolic Hypertension (ISH) with the two described methods described above. ISH is characterized by SBP value higher than $140 \mathrm{mmHg}$ and DBP value lower than $90 \mathrm{mmHg}$. ISH is frequently associated with decreased arterial compliance. Decreased arterial compliance has been associated with increased errors in single cuff BP devices [8]. The single cuff method identified ISH in 9 tests (17\% of the 52 tests) and the dual cuff method identified ISH in 19 tests (37\% of 52 tests). This difference can be attributed to lower DBP values with the dual cuff method and to inaccuracies inherent in the single cuff method. Mean value of W-MAP is 3 mmHg higher than the mean value of A-MAP. The higher W-MAP values are in better agreement with the improved multiplier 0.41 developed by Meany [7].

This study is the last of four studies that describe the novel dual cuff method for improved noninvasive determination of blood pressure. The dual cuff method is based on physiology rather than on unreliable statistical estimate as is the case of the single cuff method [9]. The first study [10] described the determination of SBP, the second study [11] concentrated on the determination of MAP, the third study [12] described the determination of DBP, and the present study integrated the three previous studies. We have also used wrist cuff waveforms for experimental determination of hemodynamics [13].

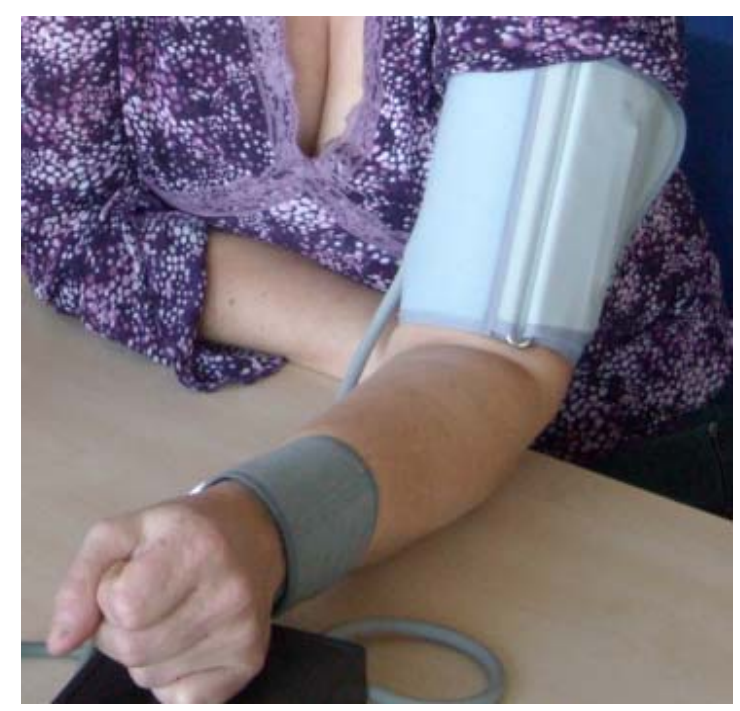

Figure 5. Example of location of arm cuff and wrist cuff during blood pressure measuring. 


\section{REFERENCES}

[1] J Jilek and T Fukushima, "Oscillometric Blood Pressure Measurement: The Methodology, Some Observations, and Suggestions", Biomed Instrum \& Technol 2005, vol. 19, pp 237-241.

[2] J Jilek and M Stork, " Pulsations in the Blood Pressure Cuff: Oscillations or Arterial Pulses?" Proc. of Int. Conf. Applied Electronics, Plzen, Czech Republic 2011.

[3] LA Geddes, "Characterization of the oscillometric method for measuring indirect blood pressure," Ann. Biomed. Eng. 1982, vol. 10, pp. 271-280.

[4] T Fujikawa, O Tochikubo, $\mathrm{T}$ Sugano, and S Umemura, "Accuracy of the pulse delay time technique with triple cuff for objective indirect blood pressure measurement." $J$ Hypertens 2013, vol. 31, pp 278-286

[5] TK Kim, YJ Chee, JS Lee, SW Nam and IY Kim, "A New Blood Pressure Measurement Using Dual-Cuffs.", Computers in Cardiology 2008, vol. 35, pp165-168.

[6] J Jilek and M Stork, "Dual Cuff System Improves Noninvasive Blood Pressure Determination", Proc. of Int. Conf. Applied Electronics, Plzen, Czech Republic 2010.

[7] E Meany, F Alva and R Moguel, "Formula and nomogram for the sphygmomanometric calculation of the mean arterial pressure", Heart 2000, Vol 84, pp. 64
[8] NM Van Popele, WJ Bos, N De Beer, D Van Der Kuip, A Hofman, and DE Grobbee, "Arterial stiffness as underlying mechanism of disagreement between an oscillometric blood pressure monitor and a sphygmomanometer," Hypertension 2000, vol. 36, pp 484-488.

[9] G S Stergiou, P Lourida, D Tzamouranis, and N M Baibas, "Unreliable oscillometric blod pressure measurement: prevalence, repeatability and characteristics of the phenomenon," J. Hum. Hypertens 2009, vol. 23, pp. 794-800.

[10] J Jilek and M Stork, "Wrist Cuff Emulates Auscultation In Dual Cuff Noninvasive Blood Pressure System", Proc of Int Conf Applied Electronics, Plzen, Czech Republic 2012.

[11] J Jilek and M Stork, "Wrist cuff method determines mean arterial pressure in dual-cuff blood pressure system", Proc of Int Conf Applied Electronics, Plzen, Czech Republic 2014.

[12] J Jilek and M Stork, "Wrist cuff method determines diastolic pressure in dual cuff blood pressure system", Proc of Int Conf Applied Electronics, Plzen, Czech Republic 2015.

[13] J Jilek and M Stork, “An Experimental System for Estimation of Blood Pressure and Hemodynamics from Oscillometric Waveforms", Proc of Int Conf Applied Electronics, Plzen, Czech Republic 2003. 